\title{
Secretory Origin and Temporal Appearance of the Porcine $\beta$-Microseminoprotein (Sperm Motility Inhibitor) in the Boar Reproductive System
}

\author{
HELLEN JENG, ${ }^{1}$ HSING-HUI CHU, ${ }^{1}$ WINSTON T.K. CHENG,${ }^{2}$ WEN-CHANG CHANG,${ }^{3,4}$ AND \\ SHUEN-JIING SU ${ }^{5} *$ \\ ${ }^{1}$ Department of Anatomy, Taipei Medical College, Taipei, Taiwan \\ ${ }^{2}$ Department of Animal Science, National Taiwan University, Taipei, Taiwan \\ ${ }^{3}$ Institute of Biochemical Sciences, National Taiwan University, Taipei, Taiwan \\ ${ }^{4}$ Institute of Biological Chemistry, Academia Sinica, Taipei, Taiwan \\ ${ }^{5}$ Department of Pathology, Po-Jen General Hospital, Taipei, Taiwan
}

\begin{abstract}
A specific antiserum against the porcine sperm motility inhibitor (SMI) was used in Western blotting analysis of tissue homogenates to reveal the possible origin of SMI in the boar reproductive system at different ages. The ages of the boar used were day 0 , day 15 , day 30 , day 60 , day 100 , day 120 , day 135 , day 150 , and day 210 . The tissue homogenates of the day 60 and older showed immunoreaction. The results were further checked by indirect immunohistochemical staining and observed under light microscope. The SMI antigen appeared in the epithelial cells and in the lumen of the secretory ducts of the prostate gland. These results indicate that porcine SMI is synthesized only by the postnatal prostate gland. The homogenate of the prostate gland of day 100 was also used for the purification of SMI. The prostatic SMI was co-eluted with the seminal SMI in the reversed phase HPLC. Mass spectrometric analysis of the prostatic SMI revealed a molecular weight of 10,066 . These results indicate that the prostatic SMI is identical to that purified from seminal plasma (Jeng et al., 1993; Biochem Biophys Res Communi 191:435-440). Mol. Reprod. Dev. 58:63-68, 2001. () 2001 Wiley-Liss, Inc.
\end{abstract}

Key Words: sperm motility inhibitor; prostate gland; Western blotting; immunohistochemical staining

\section{INTRODUCTION}

Most noncellular elements of ejaculated semen are secreted by accessory sexual glands, which comprise the seminal vesicle, the prostate gland, and the bulbourethral gland. The seminal plasma is the common secretion of accessory sexual glands and contains a wide variety of enzymatic and nonenzymatic proteins (Mann and Lutwak-Mann, 1981). A sperm motility inhibitor $(\mathrm{SMI})^{1}$ purified from porcine seminal plasma

${ }^{1}$ Since the sperm motility inhibitor (SMI) was found to be identical to $\beta$-microseminoprotein, these two terms are used interchangeably in this report. has been shown to inhibit the sperm motility in a reversible and dose-dependent manner as previously reported (Jeng et al., 1993). The amino acid sequence of the peptide fragments of SMI was determined and compared with porcine $\beta$-microseminoprotein (Fernlund et al., 1994). The agreement in the peptide sequences and molecular masses led us to conclude that SMI is identical to $\beta$-microseminoprotein ${ }^{1}$ (Chao et al., 1996). The human analog, $\beta$-microseminoprotein, also called PSP 94 (a prostatic secretory protein of 94 amino acids), is an unglycosylated protein with an unknown function (Green et al., 1990). Porcine SMI is a singlechain protein of 91 amino acids and has a deletion of three amino acids in the $\mathrm{N}$-terminal region, but otherwise manifests similarity in amino acid sequence including conservation of 10 cysteine residues of $\beta$ microseminoprotein (Fernlund et al., 1994).

Iwamoto et al. (1992) purified a motility-dynein ATPase inhibitor, named SPMI, from boar seminal plasma. Nucleotide sequence analysis of the 645-bp SPMI cDNA predicted a coded polypeptide of 137 amino acid residues, which includes a 21-residue signal peptide and a 116-residue secreted protein. SPMI is secreted by seminal vesicle (Iwamoto et al., 1993, 1995). Human seminal plasma also contains SPMI originating from the seminal vesicles as a $52 \mathrm{kDa}$ precursor form (Robert and Gagnon, 1995). Both SPMI and SMI have motility inhibitory effect on porcine sperm. However, they are not the same protein.

Our previous studies have shown that porcine SMI can inhibit the $\mathrm{Na}^{+}, \mathrm{K}^{+}$-ATPase and reduce the sperm motility reversibly (Jeng et al., 1993; Chao et al., 1996).

Grant sponsor: National Science Council; Grant number: 89-2313-B038-001.

*Correspondence to: Shuen-Jiing Su, Department of Pathology, PoJen General Hospital, Taipei, Taiwan. E-mail: acts1229@tmc.edu.tw Received 21 March 2000; Accepted 22 May 2000 
The human $\beta$-microseminoprotein was reported to be present in several mucus-associated tissues other than the reproductive organs (Weiber et al, 1990), which suggested a possible functional role in reproductive as well as nonreproductive tissues. It is of great interest to find out whether porcine $\beta$-microseminoprotein has the same distribution profile with its human analog because this information would shed some light on the problem of its biological functions. We, therefore, investigated the secretory origin of porcine $\beta$-microseminoprotein using Western blotting and immunohistochemical staining techniques. In this report, we conclude that porcine $\beta$-microseminoprotein is synthesized only by the postnatal prostate and secreted into the seminal plasma.

\section{MATERIALS AND METHODS \\ Semen Collection and Purification of Porcine $\beta$-Microseminoprotein}

The fertile boars of the MeiShan strain were used. Freshly ejaculated semen (sperm-rich fraction) was collected by the gloved-hand method into a warmed beaker and filtered through three layers of cheesecloth. The filtrate was centrifuged at $600 \mathrm{~g}$ for $30 \mathrm{~min}$ at $4^{\circ} \mathrm{C}$. The supernatant was dialyzed overnight at $4^{\circ} \mathrm{C}$ against three changes of double deionized water, lyophilized, and stored at $-70^{\circ} \mathrm{C}$ until used. The porcine $\beta$-microseminoprotein was purified from seminal plasma as previously described (Jeng et al., 1993).

\section{Antibody Induction}

A male New Zealand rabbit was used. Intrasplenic immunization was performed as previously described (Hong et al., 1989). Emulsion was prepared by mixing saline containing $0.3 \mathrm{mg}$ of antigen (SMI1) with Freund's complete adjuvant (Gibco). Two weeks after immunization, the serum titer was checked by double diffusion and solid-phase enzyme-linked immunosorbent assay (ELISA). Optical densities were read in a microplate reader (model 450, Bio-Rad). The sera with high titer were collected, aliquoted, and stored at $-20^{\circ} \mathrm{C}$.

\section{Western Blotting Analysis}

The assay of different tissue homogenates. The homogenates of porcine male reproductive organs including seminal vesicle, prostate gland, bulbourethral gland, epididymis, and testis, were prepared with 3 volumes (w/v) of extraction buffer $(50 \mathrm{mM}$ Tris- $\mathrm{HCl}$, pH 8.5, 5 mM EDTA, $100 \mu \mathrm{g} / \mathrm{ml}$ PMSF, $10 \mu \mathrm{g} / \mathrm{ml}$ leupeptin, $10 \mu \mathrm{g} / \mathrm{ml}$ pepstatin, and $10 \mu \mathrm{g} / \mathrm{ml}$ aprotinin) at $4^{\circ} \mathrm{C}$ (Kwok et al., 1993). The homogenates were then centrifuged twice at $4^{\circ} \mathrm{C}$ for $20 \mathrm{~min}$ at $20,000 \mathrm{~g}$. The supernatants were analyzed by $8-20 \%$ tricine gradient SDS-polyacrylamide gel electrophoresis (SDS-PAGE, Novex Experimental Technology) (Laemmli, 1970) and then transferred to a polyvinylidene difluoride membrane (PVDF, Immobilan-P; Millipore Corporation, Bedford). The PVDF blot was blocked with $5 \%$ skimmed milk in blocking buffer $(0.05 \mathrm{M}$ Tris- $\mathrm{HCl}, 0.9 \% \mathrm{NaCl}$, $\mathrm{pH}$ 7.6) in $4^{\circ} \mathrm{C}$ overnight. The primary antibody (the antiserum against SMI, $1500 \times$ dilution) was then incubated with the PVDF blot for $1 \mathrm{hr}$ at room temperature. After washing with TBST (Tris-buffered saline Tween buffer), 0.5 M Tris-HCl, the secondary antibody (alkaline phosphatase conjugated antibody against rabbit IgG, $2500 \times$ dilution in blocking buffer) for $1 \mathrm{hr}$. The blot was then washed and rinsed with alkaline phosphatase buffer for $5 \mathrm{~min}$. The NBT (4-nitro blue tatraedium chloride) and BCIP (5-brom-4-chlor-3-indolyl-phosphate) were added for staining. The stop solution (0.1 M Tris, 0.01M EDTA, pH 8.0) was used to stop the reaction. The blot was then air-dried. Some homogenates of other organs including trachea, stomach, duodenum, jejunum, ileum, liver, and spleen were also prepared for detecting whether they had SMI immunoreaction or not.

The assay of prostatic homogenates of different ages. The ages of the boar used were day 0 , day 15 , day 30 , day 60 , day 100 , day 120 , day 135 , day 150 , and day 210. The prostatic homogenates were applied to Western blotting analysis according to the methods described above.

\section{Indirect Immunohistochemical Staining}

Specimens were prepared by paraffin sectioning (Minotome, IEC) of the prostate gland, bulbourethral glands, seminal vesicles, epididymis, testis, trachea, stomach, duodenum, jejunum, ileum, liver, and spleen. The procedures given in the manual of the ABC detection system (DRAFT, level1, multispecies Avidin-Biotin Complex detection system; Signet Laboratories, Inc.) were followed except that some incubation times were modified. The counter-stain with hematoxylin was done. The slides were dehydrated and mounted. The control sections were treated in the same manner except that normal rabbit serum was added instead of the antiserum. These sections were observed under a microscope and photographed.

\section{Purification of the Porcine

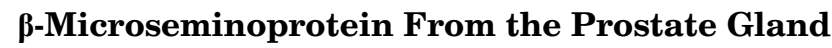

Porcine $\beta$-microseminoprotein was purified from the homogenate of the porcine prostate gland of 100-dayold pigs according to the method for the purification of SMI from seminal plasma (Jeng et al., 1993).

\section{Mass Spectrometry}

Porcine $\beta$-microseminoprotein purified from porcine prostate gland was dissolved in $50 \%$ acetonitrile containing $0.1 \%$ acetic acid to make a final concentration of $10 \mu \mathrm{M}$ and analyzed in an API-100 mass spectrometer (PE SCIEX) at an infusion rate of $5 \mu 1 / \mathrm{min}$. The spectra were analyzed by the software (BioTool Box) supplied by the manufacturer.

\section{RESULTS}

Immunohistochemical studies and Western blotting indicated that porcine $\beta$-microseminoprotein did not 


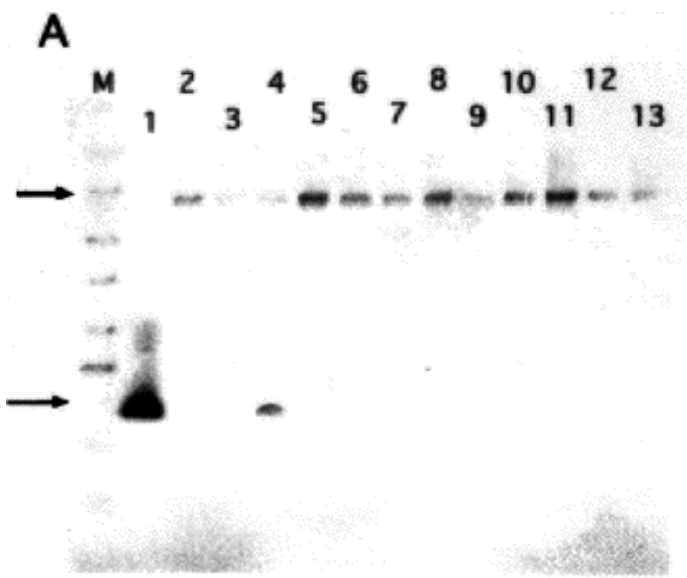

B
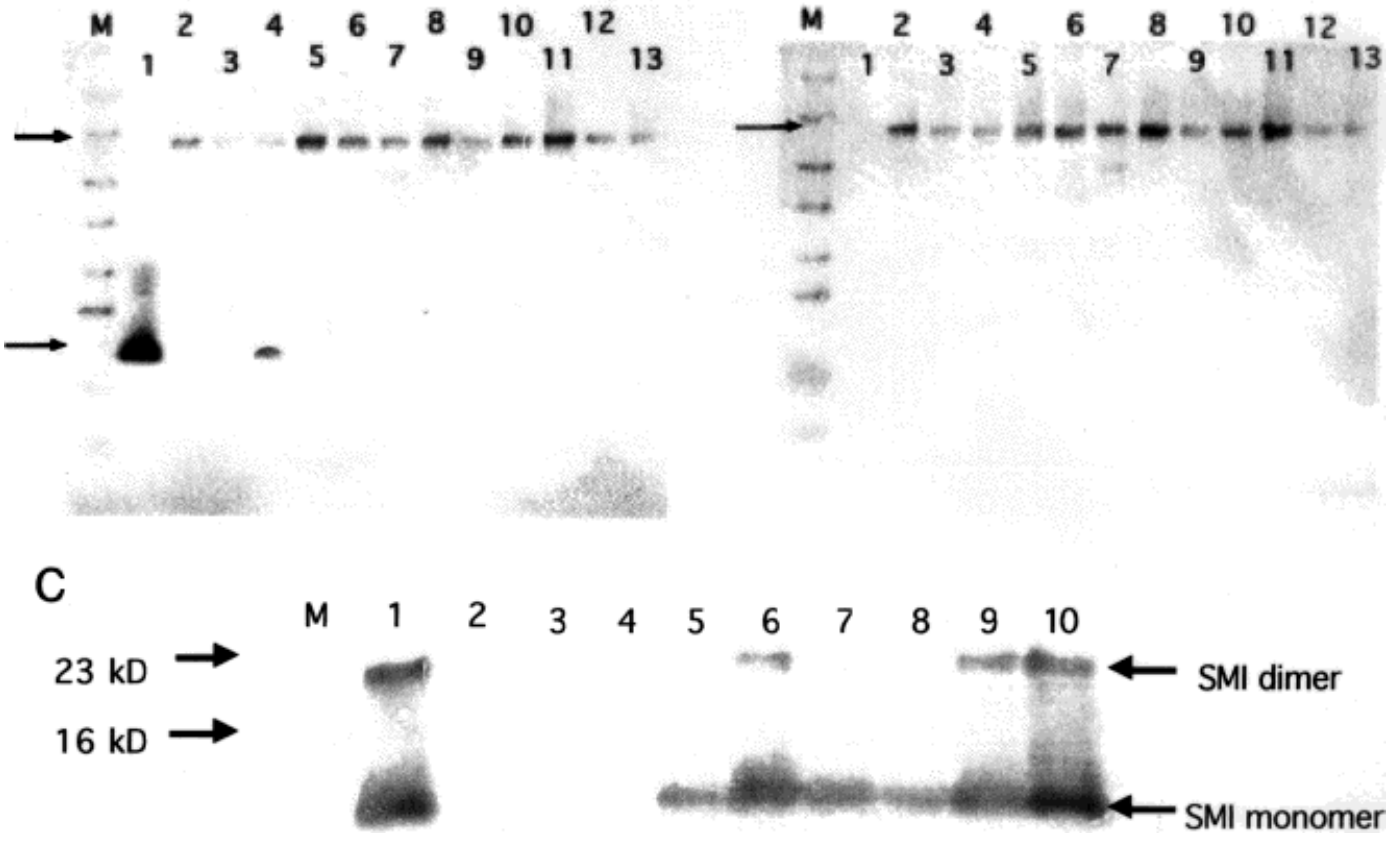

The age is 100 days. A is the experimental group; B is the control group using normal rabbit serum. The upper arrows indicate the nonspecific band of $52 \mathrm{kDa}$. The lower arrows show the position of SMI. C: temporal expression of SMI. M: molecular weight marker, 1: SMI, 2: prostatic homogenate of day 0,3 : day 15,4 : day 30,5 : day 60,6 : day 100, 7: day 120, 8: day 135, 9: day 150, 10: day 210.

appear in the prostate tissue before day 30 (Fig. 1C). At day 60 and older, it appeared only in the prostate gland (Figs. 1 and 2).

The western blotting analysis of tissue extracts (Figs. 1A, B) showed that the prostate gland is the only origin for the synthesis of porcine $\beta$-microseminoprotein. All the other tissues such as seminal vesicle, bulbourethral glands, prostate, epididymis, testis, stomach, duodenum, jejunum, ileum, trachea, spleen, and liver did not show any immunoreaction with the SMI antiserum.

The Western blotting analysis of prostatic extracts (Fig. 1C) showed that the prostate glands older than 60 days have immunoreaction with the SMI antiserum. The upper band is very likely the SMI dimer based on its immunoreaction and the molecular weight.

The secretory origin of porcine $\beta$-microseminoprotein is also confirmed as shown in Fig. 2 by light microscopy using the immunohistochemical localization method. The staining was located in the cytoplasm of the epithelial cell and within the secretory duct of prostate gland as brown pigments. The control sections incubated with normal rabbit serum show no staining except some light background in the epithelium and the seromucous gland in the submucosa of the trachea (data not shown).

Figure 3 shows the HPLC profile for the purification of porcine $\beta$-microseminoprotein. It reveals that porcine $\beta$-microseminoprotein is the major protein component of prostate gland. The molecular weight was found to be 10,066 , as determined by mass spectrometry (Fig. 4).

\section{DISCUSSION}

In our previous study (Chao et al., 1996) we found that porcine SMI is identical to $\beta$-microseminoprotein. Its human analog has been characterized in man and the ape and is one of the predominant proteins in the secretion of the human prostate gland (Lilja and Abrahamsson, 1988). The human $\beta$-microseminoprotein has been found to be present in several nonprostatic tissues by immunohistochemical staining (Weiber et al., 1990) and Northern blot analysis (Ulvsbäck et al., 1989). Its distribution profile seems to suggest some association with mucus secretion (Fernlund et al., 1996).

In the present study we found that porcine SMI was clearly detected by immunohistochemical staining of the prostate gland (Fig. 2). Other nonprostatic tissues including the seminal vesicle, bulbourethral gland, testis, and epididymis of the reproductive system; the stomach, duodenum, jejunum, and ileum of the digestive system and the trachea of the respiratory system showed very little immunoreaction. The Western blotting analysis revealed an immunoreactive band of about $52 \mathrm{kDa}$ in all tissues examined which obviously is 
A

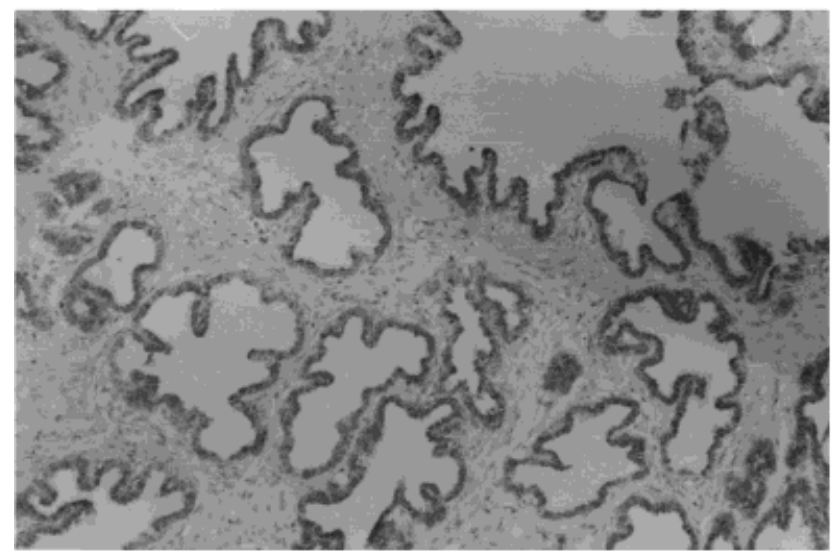

B

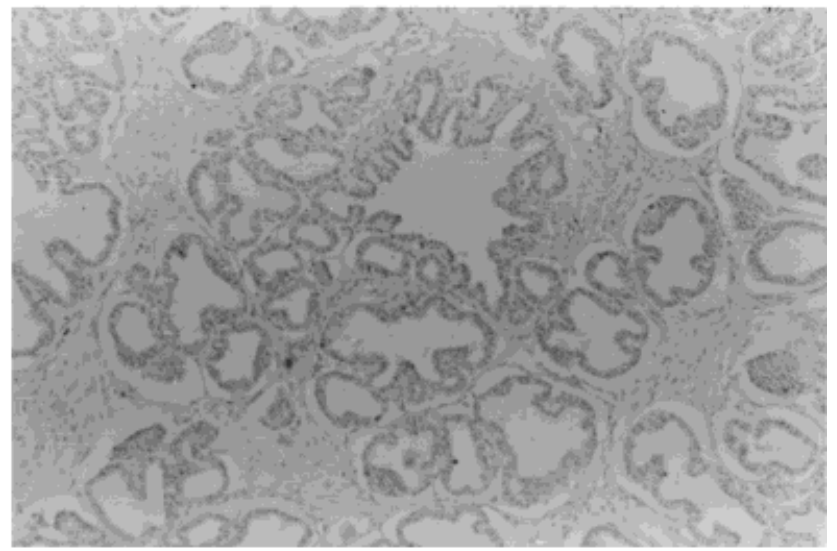

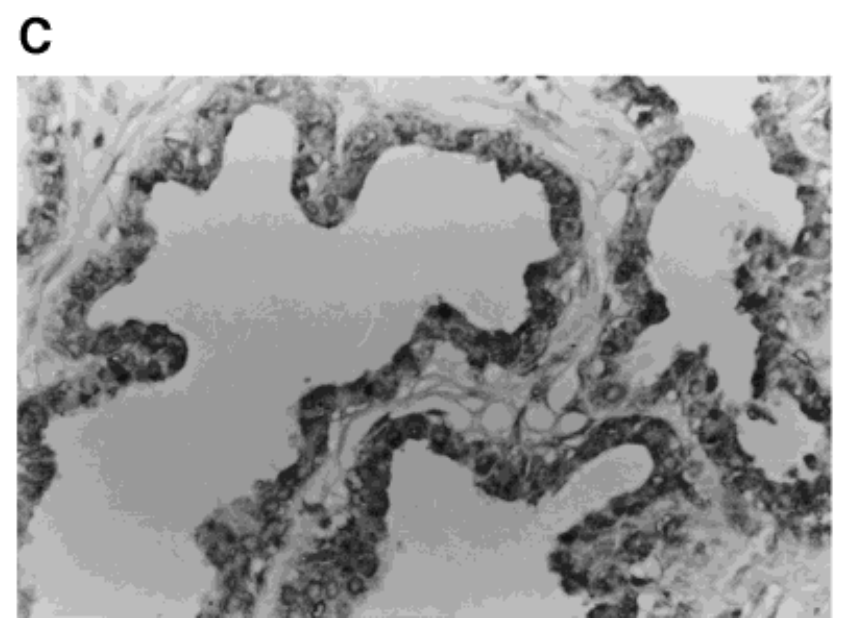

Fig. 2. A: Immunohistochemical localization of SMI in prostate gland of day 100 under a light microscope. B: Control group. C: Magnification of A. The SMI antigen is stained as brown particles in the cytoplasm of the epithelial cell within the secretory duct. A and B are $\times 100, \mathrm{C}$ is $\times 400$. [Color figure can be viewed in the online issue, which is available at www.interscience.wiley.com]

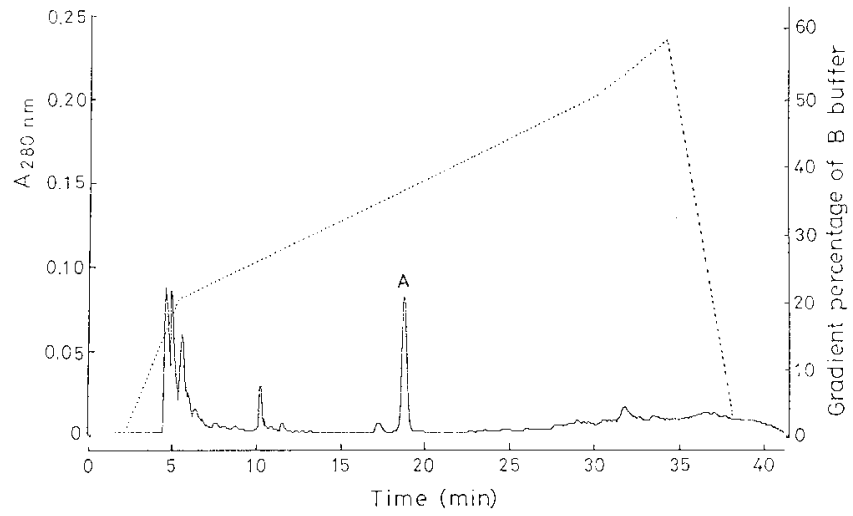

Fig. 3. HPLC profile of prostate extract through an Aquapore RP300 column. A: $0.07 \%$ trifluoroacetic acid in water; B: $0.07 \%$ trifluoroacetic acid in acetonitrile. The concentration of B increased from $20 \%$ to $60 \%$ within 30 min. Peak A is the prostatic SMI. The prostatic SMI was co-eluted with the seminal SMI in the reversed phase HPLC. Mass spectrometric analysis of the prostatic SMI revealed a molecular weight of 10,066 . not related to SMI. This immunoreactive band was also observed in the control group stained with normal serum (Fig. 1A,B). It is likely that some unknown proteins that could recognize the serum components (probably the immunoglobulins) were responsible for this nonspecific immunobinding. This positive reaction is in agreement with a very weak staining in the control group treated with normal rabbit serum (Fig. 2). The presence of $\beta$-microseminoprotein in the trachea of the pig as reported by Fernlund et al. (1994) could be due to this nonspecific immunoreaction.

The present report reveals that the human and porcine $\beta$-microseminoproteins, although homologous in sequence, are so different in its tissue distribution profile. There is no doubt that $\beta$-microseminoprotein is a prostatic protein and must have some functions in the reproductive system. Our previous report suggested one possible function of $\beta$-microseminoprotein; that is, to reversibly and mildly reduce the sperm motility which can be restored by the female follicular fluid 

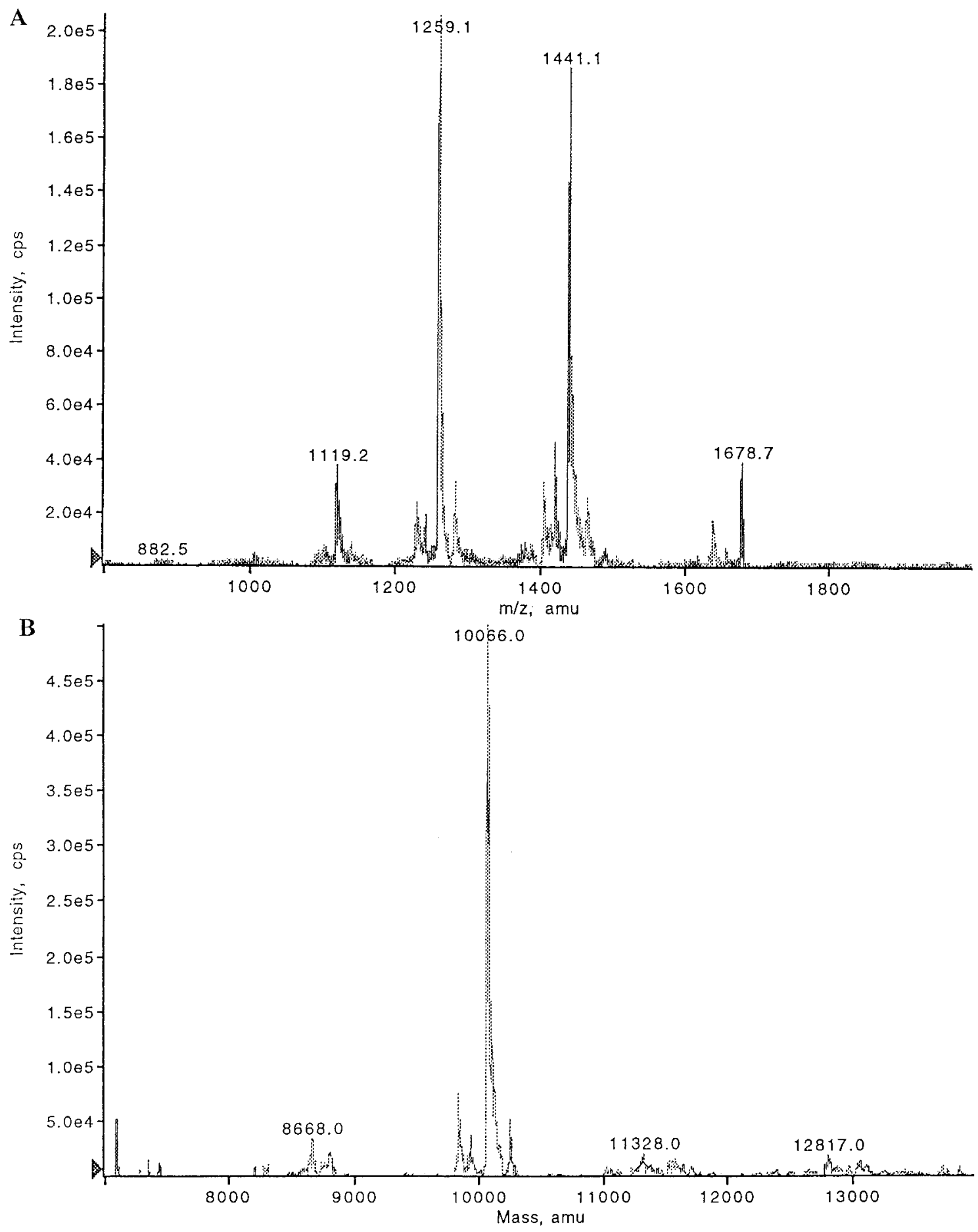

Fig. 4. Electrospray mass spectrum of the SMI purified from porcine prostate gland. A: Original spectrum. B: Computer deconvolution of the peaks in A. The theoretical value derived from protein sequence of SMI is 10,068 Da (Fernlund et al., 1994; Chao et al., 1996).

(Jeng et al., 1993). It makes sense that the sperm motility is temporarily inhibited before entering the female reproductive tract. The fact that the mature spermatids appear in the boar of MeiShan strain at 60 days of age (Cheng, 1983) is in agreement with our present finding that SMI is not synthesized until this age. All these suggest that the expression of SMI may be coordinated with the course of sexual maturation. Of course, further investigations are needed to clarify this point.

\section{ACKNOWLEDGMENT}

This work was partially supported by a grant from the National Science Council to Hellen Jeng. 


\section{REFERENCES}

Chao CF, Chiou ST, Jeng H, Chang WC. 1996. The porcine sperm motility inhibitor is identical to $\beta$-microseminoprotein and is a competitive inhibitor of $\mathrm{Na}^{+}, \mathrm{K}^{+}$-ATPase. Biochem Biophys Res Commun 218:623-628.

Cheng PL. 1983. A highly prolific pig breed of China-the Taihu pig. Pig News and Information 4:407-416.

Fernlund P, Grangerg L, Roepstor RP. 1994. Amino acid sequence of $\beta$ microseminoprotein from porcine seminal plasma. Arch Biochem Biophys 309:70-76.

Fernlund P, Granberg LS, Larsson I. 1996. Cloning of $\beta$-microseminoprotein of the rat: a rapidly evolving mucosal surface protein. Arch Biochem Biophys 334:73-82.

Green CB, Liu WY, Kwok SCM. 1990. Cloning and nucleotide sequence analysis of the human $\beta$-microseminoprotein gene. Biochem Biophys Res Commun 167:1184-1190.

Hong TH, Chen ST, Tang TK, Wang SC, Chang TH. 1989. Production of polyclonal and monoclonal antibodies in mice using novel immunization methods. J Immunol Methods 120:151-157.

Iwamoto T, Tsang A, Luterman M, Dickson J, de Lamirande E, Okuno M, Mohri H, Gangon C. 1992. Purification and characterization of a sperm motility-dynein ATPase inhibitor from boar seminal plasma. Mol Reprod Dev 31:55-62.

Iwamoto T, Tanaka H, Osada T, Shinagawa T, Osamura Y, Gagnon C. 1993. Origin of a sperm motility inhibitor from boar seminal plasma. Mol Reprod Dev 36:475-481.

Iwamoto T, Tanaka H, Osada T, Shinagawa T, Osamura Y, Gangon C. 1995. Cloning of boar SPMI gene which is expressed specifically in seminal vesicle and codes for a sperm motility inhibitor protein FEBE Lett 368:420-424.

Jeng H, Chang WC. 1997. Porcine sperm motility inhibitors reduce the acrosome reaction in boar sperm. J Chinese Soc Vet Sci 23(3) 265-268.

Jeng H, Liu KM, Chang WC. 1993. Purification and characterization of reversible sperm motility inhibitors from porcine seminal plasma Biochem Biophys Res Commun 191:435-440.

Kwok SCM, Soares MJ, McMurtry JP, Yurewicz EC. 1993. Binding characteristics and immunolocalization of porcine seminal protein, PSP-I. Mol Reprod Dev 35:244-250.

Laemmli UK. 1970. Cleavage of structural proteins during the assembly of the head bacteriophage T4. Nature 227:680-685.

Lilja H, Abrahamsson PA. 1988. Three predominant proteins secreted by the human prostate gland. Prostate 12:29-38.

Mann T, Lutwak-Mann C. 1981. In: Male reproductive function and semen. Berlin, Heidelberg, New York: Springer-Verlag. p. 269-336.

Robert M, Gagnon C. 1995. Sperm motility inhibitor from human seminal plasma: association with semen coagulum. Hum Reprod 10:2192-2197.

Ulvsbäck M, Lindsröm C, Weiber $\mathrm{H}$, Abrahamsson PA, Lilja $\mathrm{H}$, Lundwall A. 1989. Molecular cloning of a small prostate protein, known as $\beta$-microseminoprotein, PSP 94 or beta-inhibin, and demonstration of transcripts in non-genital tissues. Biochem Biophys Res Commun 164:1310-1315.

Weiber H, Anderson C, Murne A, Rannevik G, Lindsrom C, Lilja H, Fernlund P. 1990. $\beta$-Microseminoprotein is not a prostate-specific protein. Am J Pathol 137:593-603. 